\title{
Marchés énergétiques en transition : le cas de la Belgique
}

\author{
P. Agrell et A. Gautier
}

Les marchés énergétiques se sont considérablement transformés ces dernières années $\mathrm{du}$ double fait de la libéralisation des marchés et de la transition vers un mix énergétique moins carboné. La libéralisation des marchés de l'électricité et du gaz a bouleversé la chaine de production en séparant les activités concurrentielles (production, approvisionnement, commercialisation) des activités réseaux qui restent monopolistiques et régulées. L'émergence des énergies renouvelables a modifié la manière dont les consommateurs et les producteurs interagissent sur le marché du fait de leur caractère intermittent et de leur position décentralisée sur le réseau. Le modèle traditionnel vertical -production, transport, consommation- doit être complètement repensé.

En Belgique, les questions énergétiques sont au centre du débat politique et économique. De nombreux thèmes sont abordés : le coût élevé de l'énergie et son impact sur la compétitivité, le prolongement des centrales nucléaires, le blackout ou le coût des énergies renouvelables, pour en citer quelques uns. Ce numéro de Reflets et perspectives de la vie économique a pour ambition de contribuer à ce débat en proposant le point de vue de plusieurs experts sur la problématique des marchés énergétiques en transition avec chaque fois un focus particulier sur notre pays.

Les deux premiers articles de ce volumes, écrits par des experts du bureau du plan D. Devogelaer et D. Gusbin, envisagent l'évolution du mix énergétique à l'horizon 2050 en considérant des objectifs climatiques ambitieux. Ces articles mettent en avant les défis que représente la transition énergétique avec notamment des investissements conséquents à réaliser dans le domaine de la production et une diminution de la sécurité d'approvisionnement dans le nouveau mix énergétique envisagé. La nécessité d'investir dans la production, que le débat de cet hiver sur le blackout a illustré, est analysée par A. Estache et A.-S. Steichen qui calculent le coût du capital pour différentes technologies de production. Si ces auteurs mettent en avant un coût du capital plus faible pour le renouvelable, ce contexte favorable à l'investissement pourrait être remis en cause par une politique hésitante et un soutien moindre à la production d'énergie renouvelable. V. Daxbek et A. Estache comparent les avantages fiscaux dont bénéficient les entreprises du secteur de l'énergie. Ils constatent des taux de taxation effectifs faibles, ce qui constitue un élément favorable à l'investissement, mais au prix d'un mécanisme fiscal complexe. Par ailleurs, ils ne constatent pas de différence entre les avantages fiscaux par filière de production, la production renouvelable ne bénéficiant pas d'avantages supplémentaires. Par contre, ils constatent des différences sensibles entre le taux de taxation effectif des différentes composantes de la chaine d'approvisionnement.

Le soutien aux énergies renouvelables est analysé dans les articles de T. Bauwens, $\mathrm{N}$. Boccard \& A. Gautier et L. Ovaere \& S. Proost sous trois angles différents. T. Bauwens s'intéresse au soutien des consommateurs et à l'acceptabilité de l'éolien. Il montre que la propriété coopérative des éoliennes soulève de moindres résistances à l'installation de celles-ci. N. Boccard \& A. Gautier étudient le soutien financier dont l'énergie verte a bénéficié en Wallonie et ils mettent en avant le coût financier important au regard des objectifs poursuivis. Pour ce faire, ils calculent le coût de la tonne de CO2 évitée par les 
énergies renouvelables. Ovaere \& Proost se focalisent sur le soutien politique aux énergies renouvelables et mettent en avant l'hypothèse que ce soutien pourrait avoir des motivations électoralistes.

Dans le marché libéralisé, la distribution reste régulée. En Belgique, cette compétence vient d'être transférée aux régions, l'occasion pour elles de repenser la régulation. Cette question est abordée dans quatre articles. Celui de P. Agrell revient de manière détaillée sur les objectifs et les méthodes de la régulation incitative, $\mathrm{M}$. Bougnouch complète cette analyse théorique et montre que les pratiques régulatrices en Europe sont très diverses. Il compare également la régulation mise en place en Wallonie avec les pays voisins. Les contributions de J. Bouckaert et P. Agrell \& J. Teusch analysent les réformes possibles en Belgique. P. Agrell \& J. Teusch regardent les obstacles à la mise en place d'un modèle de régulation incitatif utilisant la comparaison des performances (benchmarking) en Belgique. J. Bouckaert compare la régulation dans les trois régions et regrette que la Flandre qui a proposé une nouvelle méthodologie n'ait pas complètement abandonné le (R)ROI.

La régulation consiste d'une part à mesurer les coûts de la distribution et, d'autre part, à imputer ces coûts aux consommateurs. Le tarif de distribution représente une grande partie de la facture des clients (aux alentours de 40\%). A. Gautier discute de la structure tarifaire de la distribution et met en avant l'inadéquation de la tarification au $\mathrm{kWh}$ avec l'évolution des modes de production et de consommation. Ces évolutions sont étudiées par A. Latiers qui estime le potentiel pour une gestion active de la demande en Belgique.

Le volume se conclut par deux articles qui analysent l'évolution des prix de l'énergie en Belgique. L'article de Bruynoghe et al. analyse la contribution des prix de l'énergie à l'inflation. Elle montre que les prix en Belgique sont plus volatiles que chez nos voisins mais qu'en moyenne l'augmentation des prix a été moindre. La contribution de J. van der Linden analyse plus en détail l'évolution des prix du gaz et de l'électricité en se focalisant sur les clients professionnels. Il constate une convergence et une position favorable de la Belgique pour la composante énergie mais il existe des différences sensibles en ce qui concernent les composantes réseaux et les surcharges.

Ce volume contient 15 contributions originales et de qualité. Nous espérons que cet éclairage scientifique sur les questions énergétiques en Belgique contribuera au débat politique et économique. 